\title{
Caracterización epidemiológica de las infecciones asociadas a la atención en salud en una IPS privada. Neiva 2013
}

\author{
Epidemiological characterisation of hospital-acquired infections in a \\ private hospital in Neiva in 2013
}

Juan D. Fierro', María A. Naranjo', Carolina Cabrera', Jorge Ramos²

\begin{abstract}
Resumen
Introducción: Las infecciones Asociadas a la Atención en Salud (IAAS) se han convertido en un problema de salud pública; en países desarrollados, entre el 5 y el $10 \%$ de los pacientes contraen una o más infecciones, y se considera que entre el 15 y el $40 \%$ de los pacientes internados en atención crítica resultan afectados. Métodos: Se realizó un estudio de corte transversal con enfoque analítico, con el objetivo de realizar una caracterización epidemiológica de las IAAS en una IPS privada de la Ciudad de Neiva en el año 2013. Se incluyeron 251 casos confirmados. Resultados: se logró determinar que las infecciones de mayor prevalencia fueron secundarias a procedimientos quirúrgicos (ISO), especialmente las de tipo superficial (31.9\%); seguido se encuentran las Infecciones asociadas a dispositivos médicos, con mayor proporción las del tracto urinario asociado a catéter vesical. Las neumonías asociadas a la atención en salud representaron el 14,3\%.Se tuvo una tasa de letalidad de las IAAS del 9,5\% siendo la neumonía y las infecciones del torrente sanguíneo las de mayor proporción. Se tuvo una tasa de 1,9\%. Conclusión: las características sociodemográficas de los casos de IAAS son similares al comportamiento de las instituciones de salud a nivel nacional y de países en vía de desarrollo.
\end{abstract}

Palabras clave: infección asociada a la atención en salud (IAAS), infección asociada a dispositivo médico, infección del sitio operatorio (ISO).

\begin{abstract}
Introduction: Hospital-acquired infections (HAI) have become a public health issue. In developed countries between 5 and $10 \%$ of patients contract one or more of these infections and it is believed that between 15 and $40 \%$ of patients in critical care units are affected. Method: A cross sectional study with an analytical approach was carried out in order to characterise the epidemiological features of HAls in a private hospital in Neiva in 2013. The study included 251 confirmed cases. Results: The study determined that infections were most commonly a result of surgical procedures (SSI), particularly superficial surgical procedures $(31.9 \%)$. The second major cause of infections were infections associated with medical devices, predominantly urinary tract infections associated with catheters. Pneumonia associated with health care attention represented $14.3 \%$. The fatality rate of HAls was $9.5 \%$; pneumonia and bloodstream infections accounted for the highest proportion of fatalities. Had a rate of $1.9 \%$. Conclusion: The sociodemographic
\end{abstract}

1. MD. Epidemiólogo. Universidad Surcolombiana de Neiva.

2. Enf. Epidemiólogo, Línea de Bioestadística. Grupo Infecto-Control. Docente Universidad Surcolombiana. Doctorando en Salud Pública, Universidad CES, Medellín, Colombia.

Correspondencia: Jorge Ramos. Correo electrónico: jormos2806@gmail.com

Recibido: 04/05/2014-Revisado: 13/07/2014-Aceptado: 01/08/2015 
characteristics of cases of HAls are similar to the performance of health institutions at a national level and that of developing countries.

Key words: hospital-acquired infection ( $\mathrm{HAl})$, infections associated with medical devices, surgical site infections (SSI).

\section{Introducción}

Las infecciones Asociadas a la Atención en Salud (IAAS) se han convertido en un desafío para las instituciones y los profesionales de la salud, y una necesidad de crear políticas de calidad para disminuir los costos sociales y económicos que estas suponen para el paciente, su familia y para las instituciones prestadoras de servicios de salud.

En paises desarrollados, entre el 5 y $10 \%$ de los pacientes contrae una o más infecciones y se considera que entre el 15 y el $40 \%$ de los pacientes internados en atención crítica resultan afectados ${ }^{1}$. En entornos de bajos recursos, las tasas de infección pueden superar el $20 \%{ }^{2}$, pero los datos disponibles son insuficientes para evaluar el peso de la enfermedad en los países en vía de desarrollo.

En Colombia el Grupo Nacional de Vigilancia Epidemiológica de las Unidades de Cuidados Intensivos en el 2007 reportó que las infecciones más frecuentes son las neumonías asociadas a la ventilación mecánica, infecciones del tracto urinario y bacteriemia asociada a catéter venoso central.

La I.P.S. privada de la ciudad de Neiva, es una clínica de segundo y tereer nivel de atención, localizada al sur de Colombia; cuenta con 24 camas de hospitalización pediátrica, 72 camas de hospitalización adultos, cinco camas de cuidado intermedio neonatal, una cama de cuidado intermedio pediátrico, una cama de cuidado intensivo pediátrico, seis camas de cuidado intensivo adultos, cuatro quirófanos y una sala de partos.

La incidencia de infección intrahospitalaria en los últimos años se ha incrementado debido al mejoramiento de la notificación y búsqueda activa de casos realizado por el comité de vigilancia. Actualmente en la clínica se detectan alrededor del $100 \%$ de los casos mediante notificación de los servicios por medio de fichas de inicio o cambio de esquemas de antibióticos, kardex médicos y revisión de historias clínicas. En 2009 la tasa global de infección intrahospitalaria fue del 2,1x100 egresos, en el 2010 1,8 x 100 egresos, en el 2011 1,5 x 100 egresos y en el 2012 fue de 3,3 x 100 egresos de acuerdo a la Corporación I.P.S. SaludCoop Comité de infecciones. Sin embargo, no existen estudios que muestren el impacto de este tipo de infecciones en la población hospitalaria de esta clínica de Neiva, las características asociadas a la presentación de la misma, ni la etiología de las IAAS.

Este estudio permitirá conocer las características de las IAAS de la institución permitirá ajustar las guías de manejo según los perfiles microbiológicos, restringir el uso de antibióticos según los perfiles de resistencia, conocer los factores asociados a la multirresistencia, implementar estrategias que involucren tanto al paciente, la familia y las institución de salud, establecer indicadores epidemiológicos básicos que describan la magnitud y el comportamiento de las misma que permitan compararlas con otras regiones del país y estudiar hipótesis causales o de asociación.

\section{Materiales y métodos}

Se realizó un estudio descriptivo de tipo transversal con enfoque analítico, en pacientes con IAAS durante el año 2013; la información se recolectó mediante la técnica de revisión de historias clínicas.

La población objeto fue 251 casos de IAAS y corresponden a los pacientes hospitalizados y que presentaron una infección durante su hospitalización teniendo en cuenta los criterios del Centro de Enfermedad de Atlanta (CDC). Se elaboró un formulario según las variables a estudiar, que permitiera obtener la información necesaria y fue diligenciado directamente por los investigadores.

La validez del instrumento la realizó un experto en enfermedades infecciosas y en epidemiología. La prueba piloto se realizó aplicando el formulario a diez historias clínicas escogidas al azar, con el fin de valorar la confiabilidad de este y para detectar posibles sesgos de medición y poder corregirlos.

El estudio no implicó riesgos éticos, fue revisado y aprobado por el comité de ética institucional.

Para el procesamiento de la información se utilizó una codificación establecida por el grupo investigador y la tabulación se realizó mediante tablas y gráficos estadísticos utilizando el programa SPSS Statistics 19.

Se realizó análisis univariado realizando la descripción por proporciones en las variables categóricas, y se hizo uso de las medidas de tendencia central (promedio o mediana) y de dispersión (desviación estándar y rango) para las variables numéricas; previamente se verificó el comportamiento normal de ellas mediante la prueba estadística Shapiro Wilk. Se realizó análisis bivariado de la siguiente manera; para variables categóricas se utilizó la prueba estadística Chi cuadrado $\left(\mathrm{x}^{2}\right)$ con una confiabilidad del $95 \%$. En aquellas tablas que el valor esperado sea menor a cinco, se aplicó la corrección de Yates. Finalmente se realizó un análisis de muestras independientes (Infecciones más frecuentes) con variables numéricas para identificar diferencias en las medianas utilizando la prueba estadística Kruskal - Wallis. 
Caracterización epidemiológica de las infecciones asociadas a la atención en salud en una IPS privada. Neiva 2013
R.F.S Revista Facultad de Salud

Julio-Diciembre de 2015;7(2):29-34

\section{Resultados}

El presente estudio incluye el análisis de 251 casos de Infecciones Intrahospitalarias en pacientes hospitalizados en una IPS privada en la ciudad de Neiva.

Se obtuvo un índice de masculinidad de 2,15 lo que significa que por cada hombre con IAAS se encontraron 2 mujeres infectadas. La mitad de los Pacientes fueron mayores de 50 años. La mayoría de las infecciones se presentaron en los meses de Enero (11,6\%), Febrero (12\%) y Julio $(10,8 \%)$ (Tabla 1).

Se encontró que el $44.7 \%$ de las infecciones $(n=112)$ fueron secundarias a procedimientos quirúrgicos (ISO), especialmente las de tipo superficial $(31,9 \%)$; así mismo las Infecciones asociadas a dispositivos médicos se presentaron en el 20,4\%, siendo las del tracto urinario asociado a catéter

Tabla 1. Características sociodemográficas.

\begin{tabular}{ll}
\hline Datos sociodemográficos & No. $(\%)$ \\
\hline Muestra & 251 \\
\hline Mes & \\
Enero & $29(11,6 \%)$ \\
Febrero & $30(12 \%)$ \\
Marzo & $19(7,6 \%)$ \\
Abril & $14(5,6 \%)$ \\
Mayo & $20(8 \%)$ \\
Junio & $20(8 \%)$ \\
Julio & $27(10,8 \%)$ \\
Agosto & $16(6,4 \%)$ \\
Septiembre & $17(6,8 \%)$ \\
Octubre & $25(10 \%)$ \\
Noviembre & $20(8 \%)$ \\
Diciembre & $14(5,6 \%)$ \\
EDAD (Años) Mediana (Rango) & $50(90)$ \\
\hline
\end{tabular}

\begin{tabular}{ll}
\hline EPS & \\
Cafesalud S & $16(6,4 \%)$ \\
Cafesalud C & $34(13,5 \%)$ \\
Saludcoop & $200(79,7 \%)$ \\
Particular & $1(0,4 \%)$ \\
\hline Búsqueda & \\
Ficha & $112(44,6 \%)$ \\
Calidad & $127(50,6 \%)$ \\
Antibióticos & $12(4,8 \%)$ \\
\hline Género & \\
Masculino & $80(31,8 \%)$ \\
Femenino & $171(68,12 \%)$ \\
\hline
\end{tabular}

vesical as de mayor proporción, respecto al agente etiológico, se encontró que en su mayoría son ocasionadas por gramnegativos $(14,8 \%)$; en el $37,9 \%$ de los casos no se realizó cultivo para identificación del germen y en un $11,6 \%$ su resultado fue negativo. Del total de cultivos realizados, los principales fueron sangre y orina $(30,3 \%)$; el $20,3 \%$ también se realizaron a partir de otras secreciones como líquido peritoneal, herida quirúrgica y traqueal. La mitad de los Pacientes duraron más de ocho días hospitalizados debido a su infección con un rango máximo de 47 días (Tabla 2)

Durante el año 2013 se tuvo una tasa de letalidad de las IAAS del 9,50\% siendo la neumonía y las infecciones del torrente sanguíneo las de mayor proporción $(54,17 \%)$

Tabla 2. Características de las IAAS.

\begin{tabular}{ll}
\hline Características de las IAAS & No. (\%) \\
\hline Muestra & 251 \\
\hline Tipo & \\
\hline ISO superficial & $80(31,9 \%)$ \\
Neumonía & $36(14,3 \%)$ \\
ITU & $27(10,8 \%)$ \\
ISO profunda & $18(7,2 \%)$ \\
ISO órgano espacio & $14(5,6 \%)$ \\
BACVC & $12(4,8 \%)$ \\
BACVP & $12(4,8 \%)$ \\
Traqueítis & $10(4 \%)$ \\
Otras & $42(16,7 \%)$ \\
Estancia por la IAAS (días) mediana (rango) & $8(47)$
\end{tabular}

Agente etiológico

$\begin{array}{ll}\text { P. aeruginosa } & 19(7,6 \%) \\ \text { K. pneumoniae } & 18(7,2 \%) \\ \text { E. Coli } & 9(3,6 \%) \\ \text { P. mirabilis } & 7(2,8 \%) \\ \text { S. marcense } & 5(2 \%) \\ \text { No se identificó } & 29(11,6 \%) \\ \text { No se realizó } & 95(37,9 \%) \\ \text { Otros } & 69(27,5 \%)\end{array}$

\section{Muestra cultivada}

Sangre

$50(19,9 \%)$

Orina

$26(10,4 \%)$

Herida quirúrgica

$4(1,6 \%)$

Líquido peritoneal

$4(1,6 \%)$

Secreción traqueal

$4(1,6 \%)$

Curetaje óseo

$3(1,2 \%)$

Otras secreciones 
R.F.S Revista Facultad de Salud

Julio-Diciembre de 2015;7(2):29-34

En cuanto los factores de riesgos intrínseco y extrínsecos para las IAAS, se encontró que uno de cada cuatro pacientes estaban expuestos a catéter vesical, y estancia en UCI. Más del 10\% de los infectados tenían cáncer, intubación oro traqueal, catéter central o diabetes. Finalmente, más de la mitad de los pacientes se infectaron después del día 14 de hospitalización.

Para determinar posibles factores asociados a las IAAS, el grupo investigador, decide realizar el análisis bivariado con las infecciones que representan el $70 \%$ de los casos (ITU, Neumonía, ISO). Analizando los tipos de IAAS de mayor prevalencia con los factores de riesgos intrínsecos y extrínsecos, se encontró asociación con estancia en UCI, cáncer, diabetes, catéter vesical, IOT, catéter central, traqueotomía, gastrostomía, todos ellos con significancia estadística (Tabla 3 ).

Se observa que más del $30 \%$ de los pacientes con ITU tuvieron exposición a estancia en UCI, diabetes y catéter vesical; los de neumonía a UCI y catéter vesical; y pacientes con ISO profunda a UCI, catéter vesical y catéter central. Mientras los casos de ISO superficial y profunda fueron los de menor exposición a los factores de riesgos.

Respecto a la letalidad, uno de cada cuatro pacientes con neumonía falleció, comparado con las ITU que su relación fue de 1:7. Se encontró una asociación con la mortalidad y las Infecciones del Tracto Urinario y las neumonías, siendo estadisticamente significativo $(\mathrm{P}<0,01)$.
Caracterización epidemiológica de las infecciones asociadas a la atención en salud en una IPS privada. Neiva 2013

Comparando la mortalidad de las ITU y neumonías, se encontró que después del décimo día con la infección disminuye significativamente la probabilidad de supervivencia acumulada de las neumonías respecto a las ITU. Después del décimo día, la probabilidad de supervivencia disminuye al $70 \%$ en la neumonía, mientras en la infección del tracto urinario continua con una probabilidad del $90 \%$ (Figura 1).

Se encuentra diferencia en las medianas de la edad y los días previos a la infección en las cinco IAAS de mayor prevalencia, siendo estadísticamente significativo. Donde más se observa diferencia en las medianas de edad y días previos fue en los casos de ITU con tendencia al aumento respecto a los demás casos (Tabla 4).

\section{Discusión}

El Comité de Infecciones Intrahospitalarias de la Institución Prestadora de Servicios de Salud Corporación IPS SaludCoop para el año 2013 en su registro de egresos presentó una tasa de prevalencia de $1,9 \%$, por debajo al promedio de los países en vía de desarrollo $(15,5 \%)^{3}$ y al Hospital Universitario de Neiva $(5,8 \%)^{4}$; esto se puede deber a que la institución es una entidad privada de mediano nivel de complejidad la cual ha fortalecido las estrategias de prevención de IAAS desde el año 2009.

Tabla 3. Factores de riesgo por tipo de IAAS.

\begin{tabular}{|c|c|c|c|c|c|c|}
\hline Factores & ITU & $\begin{array}{c}\text { ISO } \\
\text { superficial }\end{array}$ & $\begin{array}{c}\text { ISO } \\
\text { profunda }\end{array}$ & $\begin{array}{l}\text { ISO órgano } \\
\text { espacio }\end{array}$ & Neumonía & $X^{2}(P)^{*}$ \\
\hline Muestra & 27 & 80 & 18 & 14 & 36 & \\
\hline \multicolumn{7}{|l|}{ Factores } \\
\hline UCI & $11(40,7 \%)$ & $2(2,5 \%)$ & $0(0 \%)$ & $8(57,1 \%)$ & $11(30,6 \%)$ & $43,8(<0,01)$ \\
\hline Cáncer & $8(29,6 \%)$ & $4(5,1 \%)$ & $2(11,1 \%)$ & $2(14,3 \%)$ & $9(25 \%)$ & $14,2(<0,01)$ \\
\hline Diabetes & $9(33,3 \%)$ & $3(3,8 \%)$ & $1(5,6 \%)$ & $3(21,4 \%)$ & $8(22,2 \%)$ & $19,3(<0,01)$ \\
\hline Diálisis & $3(11,1 \%)$ & $1(1,3 \%)$ & $0(0 \%)$ & $0(0 \%)$ & $3(8,3 \%)$ & $8,8(0,07)$ \\
\hline VIH & $0(0 \%)$ & $0(0 \%)$ & $0(0 \%)$ & $0(0 \%)$ & $1(2,8 \%)$ & $3,1(0,52)$ \\
\hline Catéter vesical & $16(59,3 \%)$ & $5(6,3 \%)$ & $2(11,1 \%)$ & $8(57,1 \%)$ & $11(30,6 \%)$ & $42,5(<0,01)$ \\
\hline IOT & $5(18,5 \%)$ & $0(0 \%)$ & $0(0 \%)$ & $2(14,3 \%)$ & $4(11,1 \%)$ & $16,2(<0,01)$ \\
\hline Tubo tórax & $1(3,7 \%)$ & $1(1,3 \%)$ & $0(0 \%)$ & $0(0 \%)$ & $2(5,6 \%)$ & $3,3(0,5)$ \\
\hline Catéter central & $7(25,9 \%)$ & $1(1,3 \%)$ & $0(0 \%)$ & $5(35,7 \%)$ & $6(16,7 \%)$ & $25,8(<0,01)$ \\
\hline Traqueostomía & $4(14,8 \%)$ & $0(0 \%)$ & $0(0 \%)$ & $0(0 \%)$ & $1(2,8 \%)$ & $13,5(<0,01)$ \\
\hline Gastrostomía & $4(14,8 \%)$ & $0(0 \%)$ & $0(0 \%)$ & $0(0 \%)$ & $4(11,1 \%)$ & $15,2(<0,01)$ \\
\hline \multicolumn{7}{|l|}{ Letalidad } \\
\hline $\mathrm{Si}$ & $4(14,8 \%)$ & $0(0 \%)$ & $0(0 \%)$ & $0(0 \%)$ & $9(25 \%)$ & $27,1(<0,01)$ \\
\hline
\end{tabular}

* Prueba de Chi cuadrado con corrección de Yates. 


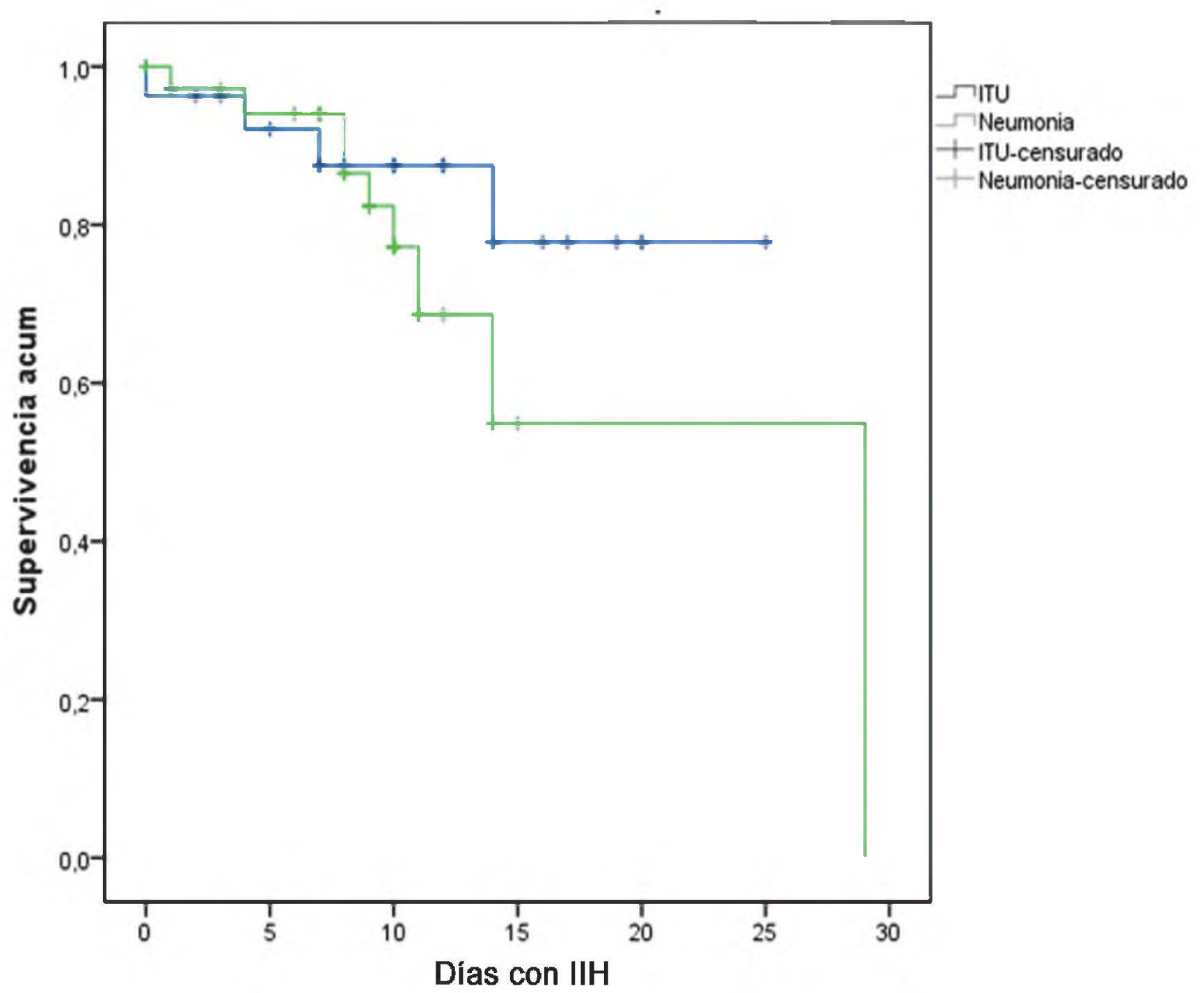

Figura 1. Supervivencia acumulada en la ITU vs. neumonía.

Tabla 4. Edad, estancia y días previos por cada tipo de IAAS.

\begin{tabular}{|c|c|c|c|c|c|c|}
\hline Factores & ITU & $\begin{array}{c}\text { ISO } \\
\text { superficial }\end{array}$ & $\begin{array}{c}\text { ISO } \\
\text { profunda }\end{array}$ & $\begin{array}{c}\text { ISO órgano } \\
\text { espacio }\end{array}$ & Neumonía & $P^{*}$ \\
\hline Muestra & 27 & 80 & 18 & 14 & 36 & \\
\hline \multicolumn{7}{|l|}{ Edad (años) } \\
\hline Mediana (rango) & $76(89)$ & $39(77,9)$ & $48,5(55)$ & $53(48)$ & $53(89)$ & $<0,01$ \\
\hline \multicolumn{7}{|c|}{ Estancia por la IIH (días) } \\
\hline Mediana (rango) & $10(25)$ & $4(43)$ & $8(47)$ & $11,5(42)$ & $9(29)$ & 0,41 \\
\hline \multicolumn{7}{|l|}{ Días previos a $\mathrm{IIH}$} \\
\hline Mediana (rango) & $22(54)$ & $10(57)$ & $15(47)$ & $8(29)$ & $9(44)$ & $<0,01$ \\
\hline
\end{tabular}


R.F.S Revista Facultad de Salud

Julio-Diciembre de 2015;7(2):29-34
Caracterización epidemiológica de las infecciones asociadas a la atención en salud en una IPS privada. Neiva 2013
Las cuatro infecciones que más se presentaron en el estudio coinciden con los datos del Centro de Enfermedades de Atlanta $\mathrm{CDC}$, donde reportan que los tipos de infecciones que más se presentan en los hospitales son ITU (34\%), ISO $(17 \%)$, BACVC (14\%) y neumonía $(13 \%)^{5}$.

La mediana de estancia por la IAAS fue de 8 días similar al comportamiento de las IPS del distrito de Bogotá$^{6}$ y cifras que no se difieren de los reportes internacionales ${ }^{5}$ que revelan la magnitud y severidad de las infecciones en los pacientes, sus familias y por supuesto en el sistema de salud.

En el $37.9 \%$ de los casos no se realizó prueba de identificación del germen porque se relaciona con los casos de ISO superficial, evento en el cual no se encuentra recomendado realizar cultivo por su baja sensibilidad ${ }^{7}$. Dentro de las infecciones con cultivo positivo, la mayoría son infecciones causadas por bacterias gramnegativos, dato similar a lo reportado en la literatura mundial y local ${ }^{8}$. Llama la atención que el microorganismo Acinetobacter baumannii causa menos del 1\% las IAAS; factor determinante positivo para evaluar la funcionalidad del comité de infecciones intrahospitalarias (IIH). En el estudio la tasa de letalidad fue inferior al promedio de países en vía de desarrollo 9 ; la principal causa de muerte fue la neumonía (25\%), seguido de la ITU (14.8\%), este último similar al promedio Nacional ${ }^{10}$.

Finalmente, en el estudio se observó que la edad y la estancia previa a la IAAS son diferentes en los cuatro tipos de infecciones más frecuentes (ISO superficial y profunda, neumonía e ITU); esto se podría deber a que los procedimientos quirúrgicos son más frecuentes en población en edades productivas y que para el desarrollo de la infección quirúrgica no influye de manera significativa la estancia previa, sino el riesgo quirúrgico (tipo de herida, duración, entre otros); mientras que las infecciones respiratorias y urinarias se presentaron en su mayoría en pacientes con edades mayores y su riesgo aumenta con cada día de hospitalización. Llama la atención que la mitad de los que tuvieron infección urinaria eran mayores de 76 años, diferente al comportamiento nacional y similar a lo reportado en Argentina, y su mediana de estancia por el evento fue inferior al promedio nacional y similar al país de México ${ }^{11}$.

La principal limitación del estudio, fue que por su diseño metodológico no se logró establecer asociación causal para las IAAS; sin embargo se analizaron la prevalencia de los factores de riesgo y se compararon de acuerdo al tipo de infección.

\section{Referencias}

1. Lazzari S, Allegranzi B, Concia E. Making hospitals safer: the need for a global strategy for infection control in healthcare settings. World Hospitals and Health Services, 2004, 32, 34, 36-42.

2. Pittet, Didier. "Infection control and quality health care in the new millenium." American journal of infection control 33.5 (2005): 258-267.

3. Rosenthal, Victor D. "Health-care-associated infections in developing countries." The Lancet 377.9761 (2011): 186-188.

4. González, Laura, et al. "Infecciones intrahospitalarias en servicios de medicina interna y cirugía del Hospital Universitario de Neiva, 2012." Revista Facultad de Salud-RFS 5.2 (2015): 27-33.

5. Department of health and human services. Action Plan to Prevent Health care - associated infections. 2009. [Internet]. Disponible en: http://www.hhs.gov/ash/ initiatives/hai/actionplan/hhs_hai_action_plan_ final_06222009.pdf

6. Secretaría Distrital de Salud de Bogotá; Boletín Epidemiológico de Infecciones Asociadas a la Atención en Salud. 2010. [Internet]. Disponible en: http:// www.saludcapital.gov.co/sitios/NigilanciaSaludPublica/ Todo\%20llH/Boletin_IIH.pdf

7. Dudeck, Margaret A., et al. "National Healthcare Safety Network (NHSN) report, data summary for 2012, Deviceassociated module." American journal of infection control 41.12 (2013): 1148.

8. Peleg, Anton Y., and David C. Hooper. "Hospitalacquired infections due to gram-negative bacteria. "New England Journal of Medicine 362.19 (2010): 18041813.

9. Rosenthal, Victor Daniel, et al. "The attributable cost, length of hospital stay, and mortality of central lineassociated bloodstream infection in intensive care departments in Argentina: a prospective, matched analysis." American journal of infection control 31.8 (2003): 475-480.

10. Rosenthal, Victor D., et al. "Time-dependent analysis of length of stay and mortality due to urinary tract infections in ten developing countries: INICC findings." Journal of Infection 62.2 (2011): 136-141.

11. Rosenthal, Victor D., et al. "Time-dependent analysis of length of stay and mortality due to urinary tract infections in ten developing countries: INICC findings." Journal of Infection 62.2 (2011): 136-141. 